\title{
The Position of the Subjectness of the Subject-less Conventional Infinitives
}

\section{Lemohang Hilda Tjabaka ${ }^{1, *}$}

${ }^{1}$ Department of English, Faculty of Humanities, National University of Lesotho, Roma 180 Lesotho

\begin{tabular}{llllll}
\hline Received: 23.05.2021 $\quad$ Accepted: 20.07.2021 & • & Published: 12.08.2021 & • Final Version: 12.08.2021
\end{tabular}

\begin{abstract}
The English conventional infinitive clauses have either overt or covert subjects. Previous analyses mainly used the PRO Theorem and Null-Case Approaches to show the distribution of the subjectness of the subject-less conventional infinitives. However, this paper investigated the position of the subjectness of the subject-less conventional infinitive clauses using the Minimalist Program's Movement Theory of Control and the External Token Merge. In this qualitative paper, data was extracted from the previous English Ph.D. research studies conducted by English native and nonnative speakers, and analysed using successive approximation analytic tools. The results demonstrate that the control and SSR-infinitives are generated in a similar fashion, which is why they share the type of subjectness. The base-generated PRO, which is claimed to be the subjectness of the control infinitives, is further revealed that is derived from movement. Therefore, for uniformity purposes, the subjectness of the control and SSR-infinitives is found to be a trace-copy ( $t$ ) or a lexical subject positioned at the [spec-IP], [spec-TP], and [spec-vP]. The analysis suggested connectivity between the structure of the subject-less conventional infinitive clause and the position of the subjectness of the subject-less conventional infinitives as the structure of the subject-less conventional infinitives informs the distribution.
\end{abstract}

Keywords: subject-less conventional infinitives, control infinitives, SSR-infinitives, subjectness, lexical subject

\section{Introduction}

Genealogically, English is the Western Germanic language brought by the first Anglo-Saxon settlers who came from Germany to Britain. Between the eighteenth and twentieth centuries, 'Britain had gained its position as the world power with many colonies all over the world' (Murtiana, 2012:109). Crystal (2003) also observes that the colonial developments continued to spread in Africa and South Pacific, which led to the expansion of English across the globe in the nineteenth century. Apart from colonialism, both geographical-historical and socio-cultural strands led to the spread of English. As a result, English has become the global language spoken by both native and non-native speakers. Lesotho being the post-protectorate of Britain is a non-native-speaking country where English is spoken as a second language. Kamwanganalu and Muyo (2003:41) note that English is mainly used as diplomacy, international business transaction, government, administration, and education.

In the education system, particularly at the tertiary level, the admitted students at the National University of Lesotho (NUL) are said to be less proficient in English. This claim is maintained by Ekanjume-Illongo (2015), who affirms that the English Department at the NUL has been complaining about the poor quality of the students they admit as far as English is concerned. The

\footnotetext{
*Corresponding Author: 1tjabaka@gmail.com
} 
students fail to construct correct sentences either written or spoken. Despite the students' majors, they are all introduced to Communication and Study Skills. In addition to this course, the Department of English offers Syntax as one of the linguistics courses. In academic and business world, syntax plays a vital role as people are expected to construct grammatically correct sentences.

As part of structural descriptions, the current paper therefore explores the subjectness of the English subject-less conventional infinitives which is also known as second/ full/ marked/ cleft/ toinfinitives within the syntactocentric perspective. Subjectness means the quality of the subject whereas subject-less is the syntactic structure that lacks a visible subject. Based on Kibort's (2006) view, subject-less constructions are the ones formed from predicates lacking overt or covert syntactic subject, and can take part in syntactic operations such as control or raising. In this regard, the conventional infinitives have two types derived from these two syntactic operations mentioned in Kibort's (2006) definition: control and raising. In accordance with Boeckx et al., (2010), the difference between control and raising lies on movement in earliest account within generative grammar. Based on this perception, movement is engaged in raising, and its subjectness is represented by trace (t) (Boeckx et al. (2010). Contrary to raising, Hornstein and Polinsky (2010) concur that control does not involve movement as it is base-generated, and its subjectness is signalled by an empty category PRO. This PRO anaphorically depends on its antecedent which is also known as its controller in the matrix clause. In addition to movement feature, Davies and Dubinsky (2004); Hornstein and Polinsky (2010) assert that for the control constructions, a determinative phrase (DP) bears more than one role, that is, it is semantically linked to both matrix and embedded verbs while with the raising constructions, the raising verb does not assign a theta-role to its subject, that is, the DP is semantically connected to the embedded verb. The control infinitive construction is provided in example (1).

(1) Lerato likes [PRO to teach Mathematics].

Example (1) represents the control infinitive clause. From this example, Lerato is interpreted as the "liker" and the "teacher"; hence the bearer of the two roles. However, to avoid violating theta criterion principle which states that a DP should bear one role, PRO is then base-generated in the embedded clause, and it is controlled by its antecedent Lerato. Consequently, the conventional infinitive clause to teach Mathematics shares the subject with the matrix clause Lerato likes. At the $\mathrm{PF}$, the conventional infinitive clause has a covert subject as it is not explicitly stated who did the action of teaching.

Raising infinitives are also reflected in example (2) and (3).

(2) The priest expected [the congregation to come].

(3) They seemed [t to enjoy his company].

Example (2) and (3) depict raising infinitive clauses. Example (2) is Subject-to-Object-Raising (SOR) which is also known as Raising-to-Object (Davies and Dubinsky, 2004; Chomsky, 2015). According to Davies and Dubinsky (2004), SOR is an NP following the matrix verb which is semantically related to only the embedded verb, and this kind of construction was initially known as exceptional case marking (ECM). The subject of the conventional infinitive clause the congregation to come appears immediately after the matrix verb, commonly known as ECM verb expected. The subject the congregation raised from the subject position to the object position to check the objective case (Radford, 1997) as the verb expected is a two-place predicate, that is, it needs two arguments. Therefore, the conventional infinitive clause in this example has the overt subject since the congregation is responsible for the action of coming. 
Looking at the example (3), it is a Subject-to-Subject-Raising (SSR) which is also called Raisingto-Subject or Noun-Raising-to-Determinative (N-Raising to D) (Davies and Dubinsky, 2004; Chomsky, 2015). SSR is an NP in a matrix clause which is semantically related to an embedded verb (Davies and Dubinsky, 2004). Following Radford (1997), subjects originate from the specifier position, also known as subject position within the VP, and subsequently raise to the specifier inflectional phrase (IP) for checking purposes. The subject they relates to the embedded verb enjoy not the matrix verb for the verb seems does not assign any theta role to the subject they. The subject they just raised to become the subject of the whole sentence. Nonetheless, at the PF, the embedded SSR-infinitive in this example lacks the overt subject.

In spite of the types of the subjectness and the types of the subject-less conventional infinitives studied by different scholars across the globe, there is a paucity of literature with regard to the position of the subjectness of the subject-less conventional infinitives. Even for the scholars who undertook a research on this phenomenon, there is a lot of controversies surrounding the distribution of the subjectness of the subject-less conventional infinitives. Therefore, the present study aims to describe the position of the subjectness of the subject-less conventional infinitives in a sentence.

\section{Literature Review}

Stemming from global literature, languages such as Polish and English have been studied. Those studies draw from various viewpoints, including hybrid, communication-and-cognition, and syntactocentric perspectives. The studies include Kučanda (2000), Nakagawa (2001), Kibort (2006), Runner (2006), and Rashid (2017). Kučanda (2000) emphasizes that to distinguish between covert and overt conventional infinitives, the type of the matrix verb should be considered.

Researchers including Kibort (2006) and Rashid (2017) investigated subject-less constructions stemming from the communication-and-cognition perspective. However, their results did not stipulate the distribution of the subjectness of the conventional infinitives which was explored by the current researcher. For inherently impersonal predicates that truly lack a syntactic subject, Kibort suggested that their morpho-lexical impersonalisation could be analyzed as having PRO for the predicate's subject. In spite of stating the type of the subjectness for the impersonal predicates that include the conventional infinitive constructions, Kibort's study has not identified the position of that subjectness. Following Davies and Dubinsky (2004) on the notion of raising and control, the present researcher assumes that not all the impersonal predicates are formed similarly; therefore, their subject may not be only PRO, but also trace (t).

Contrary to their perspective, Runner (2006) conducted a study following hybrid or eclectic perspective. In his work, Runner discussed lingering challenges to the raising object and object control. He analysed his data following both the Head-driven Phrase Structure Grammar (HPSG) and the MP as theoretical frameworks underpinning his study. The HPSG is an eclectic theory that absorbed bit and pieces from different theories including the generative grammar. However, it has not inherited the logical form (LF) and the phonetic form (PF) levels which are main syntactic levels found in the syntactocentric perspective. For this reason, the results do not articulate anything about the position of the subjectness of the subject-less conventional infinitives at the LF. Nevertheless, it suggested that there is no structural position in the embedded clause containing trace or PRO. Equally important, within the HPSG analysis, the DP functioning as an object of the matrix verb is associated with the embedded predicate by means of co-indexing called structure-sharing. However, this is not the case with the adopted theory in the current study as supported by Runner (2006) in his overt analysis. Move is one of the key operation in both overt and covert analysis. 
Using the Minimalist Program, Runner's findings however, revealed that at the LF, a determinative phrase (DP) raises from embedded clause to main clause. That is, a DP occupying an object position of the matrix verb is regarded as functional specifier of the infinitive clause. He further discovered that for the overt analysis, the thematic subject of the embedded clause is in the main clause at the LF just like the covert raising analysis. His concern was relatively on the overt subject and its position in the conventional infinitive constructions. However, it paved the way towards the distribution of the subject-less conventional infinitives in a sentence.

Different from the hybrid perspective, Nakagawa (2001) conducted a study on bare verbaliser phrase (vP) analysis of the infinitival clause in the Old English (OE) drawing from the syntactocentric perspective. Based on the Phase Impenetrability Condition (PIC), Nakagawa's (2001) results noted that PRO is located in specifier, the verbaliser phrase [Spec, vP], but not [Spec, Tense Phrase (TP)] of the OE infinitive clause as the OE infinitive clause has no T-node. According to his findings, this distribution of PRO follows an assumption that the infinitive clauses have basically the same structure as finite clauses. Looking at the infinitives starting from the Middle English (ME), Nakagawa (2001) revealed that traditionally, PRO is positioned in [Spec, TP] as the null case is checked in the specifier position of T, but in his paper, PRO appears between Inflectional Phrase (IP) and verb bar (V). There is a similitude between what Nakagawa did in his study and the current one. The two studies concentrate on the position of the subjectness of the subject-less conventional infinitives. However, there is a relative difference from his study. The present one adopted the MP's Movement Theory of Control (MTC) and External Token Merge (ETM) to identify and describe the position and type of the subjectness subject-less conventional infinitives.

From the review of related literature, it was observed that the studies drawing mainly from other perspectives other than the syntactocentric perspective hardly articulated the type and the position of the subjectness of the subject-less conventional infinitives explicitly. Despite the previous researchers' perceptions on the type of the subjectness of the conventional infinitives, they have not identified the position of the subjectness of the subject-less conventional infinitives starting from Middle English to the present. The present researcher believes that the perspectives, as well as the theories adopted in earlier research studies, influence the results on distribution and the type of the subjectness of the subject-less conventional infinitives. Even those who espoused similar perspective and theory as the current study, the researcher still anticipates different reflection as some of the concepts such as the Phase Impenetrability Condition (PIC) in Nakagawa's study are not adopted in the present paper. Another question that remains is: Does the contemporary analysis still relies on two syntactic operations: raising and control? For this reason, the present study adopted the Minimalist Program to analyse the position of the subjectness of the subject-less conventional infinitives.

\subsection{Theoretical Framework}

The paper is underpinned by the Minimalist Program (MP) which is also known as Minimalism. Minimalism is the theory proposed by Chomsky (1993). To describe the position of the subjectness of the subject-less conventional infinitives, the MP's Movement Theory of Control (MTC), External Token Merge (ETM) and VP-Internal Subject Hypothesis (VISH) were adopted. Within the MP, movement of lexical elements is permissible whenever it is necessary. As a result, the MP's MTC stipulates that the subjectness of the subject-less conventional infinitives, in particular, PRO which is the null subject for the control infinitives is analysed the same way as the raising construction which is movement. For this reason, the present study is interested in the PRO-free version of the 
MTC as it assumes movement of PRO in a sentence (Hornstein \& Polinsky, 2010; AlTameemy \& Alrefaee, 2021).

The MTC is coupled with operation copy and merge together with VISH as they allow some objects, in particular, A-movement (DP) to be re-merged and leave the copy behind. Operations copy and merge assume that the movement leaves behind copy of moved and re-merged element instead of replacing it by an indexed trace (Al-Horais, 2013). The researcher also used the ETM which postulates that instead of moving a syntactic element, another instance, that is, token of that element from the array can be driven by interface necessity and be merged externally (Krivochen, 2012). According to Krivochen (2012), there is no restriction on the number of token to be merged in structural positions that leads to optimally relevant interpretations in both interfaces, hence the elimination of sub-indexes.

\section{Materials and Methods}

In this qualitative paper, the researcher employed a case study design. A case study is observed to be one of the qualitative methods used in applied linguistics (Riazi, 2016). As a result, the researcher did an in-depth investigation to describe the position of the subjectness of the subject-less conventional infinitive in a sentence. Just like Omo and Destiny (2020) who extracted data from documents, the current study also collected data from documents, in particular, the English $\mathrm{PhD}$ studies carried out by both English native and non-native speakers. On the basis of nativism, these documents were used jointly as "empiricism can be made more amenable to the nativist" (Kinsella, 2009:5). In spite of the authors' nativism, an implication is that they are widely exposed to English language and linguistics starting from undergraduate to postgraduate, specifically $\mathrm{PhD}$ level. This language is also their specialisation. The authors of these documents are therefore assumed to be at more advanced stage of structural competence which resulted in knowledge and experience on how to construct the subject-less conventional infinitives than diploma, first degree and Master's degree holders. The documents were conveniently and purposively selected. The successive approximation analytic tool was used to analyse data. Neuman (2013:489) states that successive approximation is a "qualitative analytic tool that includes making cyclic interaction with empirical data and the abstract concepts, theories or modes, adjusting theory and refining the collected data from time to time."

\section{Results}

From the evidence in the current study, the researcher presents the following as the findings:

- The control infinitives, as well as the subject-to-subject infinitives (SSR-infinitives), are subject-less conventional infinitive constructions as they have covert subjects.

- The distribution of the subjectness of the control infinitives and the SSR-infinitives is the same.

- Even if the subjectness of the control infinitives was initially regarded as PRO that is basegenerated, the results in the present study show that PRO is formed in a similar fashion with trace which is through movement.

- The study also found that the control infinitives and the SSR-infinitives can be a trace-copy (t) or a lexical subject. 
- Lastly, this study discovered that the subjectness of the subject-less conventional infinitives is positioned at the [spec-IP], [spec-TP] and [spec-vP].

\section{Discussion}

The researcher aimed at describing the subjectness of the subject-less conventional infinitives in relation to its distribution. In order to describe the position of the subjectness of the subject-less conventional infinitives, she used the VP-Internal Subject Hypothesis (VISH), the Movement Theory of Control (MTC) and the External Token Merge (ETM). Consequently, this section is divided into three sub-divisions: VP-Internal Subject Hypothesis, Movement Theory of Control, and External Token Merge. It is worth noting that the VISH is applied throughout the analysis in this paper.

\subsection{VP-Internal Subject Hypothesis}

VP-Internal Subject Hypothesis, also known as Predicate Internal Subject Hypothesis (PISH) is the hypothesis claiming that subjects originate internally within the VP having an appropriate verb (Radford, 2004; 2009). The data used shows that the distribution of the subjectness of the subjectless conventional infinitives either control or subject-to-subject raising infinitives is the same. What differs is the type of the subjectness. This view is confirmed by Runner (2006) that there is a structural position in the embedded clause containing either PRO or trace $(\mathrm{t})$. Therefore, this paper departs from the claim made by Wurmbrand (1998) and Hornstein \& Polinsky (2010), which states that the positioning of the subjectness of raising infinitives differs from that of control infinitive even though they are somehow the same. Thus, the current study reflects that the distribution of the subjectness of both raising and control infinitives is similar as illustrated in example (4) and (5) respectively, and the structure is adapted from Radford, 2004.

(4) [TP [D $I_{i} \mathrm{VP}\left[\mathrm{V}\right.$ attempt TP- $\mathrm{DP} P \boldsymbol{P O}_{i}\left[\mathrm{~T}\right.$ to $\mathrm{VP}\left[\mathrm{DP} P R O_{i}[\mathrm{~V}\right.$ answer [DP these questions]]][]]]]

Example (4) presents the position of the subjectness of the control infinitive I attempt to answer these questions. From this example, the finding reflects that the subjectness of the control infinitive embedding is at the lower [spec-TP]. This finding is supported by Nakagawa's (2001) observation that traditionally, PRO was positioned in [spec-TP] as null case is checked in the specifier position of T. Based on the VISH, the subject $\boldsymbol{I}$ in the higher [spec-TP] originates from the [spec-VP] I answer these questions as the verb answer is a two-place predicate which has an agent to engage in an action of answering and something to be answered. Through short move and re-merging, the specifier $I$ moves from the [spec-VP] leaving its copy in a form of PRO, and re-merges with the T-bar to satisfy tense feature carried by control infinitive-to. This copy, also known to be a controlee is signalled by $\boldsymbol{P R O}$. The control verb is attempt while the controller is the subject $\boldsymbol{I}$. The specifier $\boldsymbol{I}$ then moves to the higher [spec-TP] leaving another copy, PRO to check T-feature carried by finite T. In addition to the T-feature, the Extended Projection Principle (EPP) carried by finite T $\varnothing$ enables it to attract $I$ to become its specifier forming the TP I attempt PRO to PRO answer these questions at the LF. However, at the PF, these PROs are deleted becoming TP I attempt to answer these questions. Control infinitives are said to carry tense features; so, they need to be satisfied. The symbol $/ \boldsymbol{i} / \mathrm{placed}$ next to the specifiers represents index to show that there is a connection between syntactic elements. In the case of example (4), there is a connection between the [spec-VP], the lower [spec-TP] and the higher [spec-TP]. 
The movement of the D from example (4) is known to be successive-cyclic (Radford, 2009) because I becomes the specifier of the $\overline{\mathrm{V}}$ answer these questions before moving to the embedded clause $\mathrm{T}$ constituent to and from the T constituent to before further moving to the higher [spec-TP] to become the specifier of the main clause $\mathrm{T}$ constituent which has $\emptyset$. It should be noted that this movement engaged in this sentence is the shortest movement to satisfy economy consideration (Kinsella, 2009).

Correspondingly, the SSR-infinitive's subjectness appears in the same position as the control infinitives, as shown in example (5).

(5) [TP [D To-infinitives S $_{i}$ VP [V appear TP [D $t_{i}$ [T to-AUXP [D $t_{i}$ [AUX be VP [D $t_{i}$ [V answering [DP the questions]]]]]]]]] (The hierarchy is taken from Radford, 2004)

Example (5) shows the structure that reflects the subjectness of the SSR-infinitive To-infinitive appears to be answering the questions. Unlike the previous researchers such as Runner's (2006) who did not clearly stated the distribution of the subjectness of the subject-less conventional infinitives, the present paper identifies the position of the subjectness of the SSR-infinitive as the [spec-TP]. According to Hornstein \& Polinsky (2010), the subjectness of the SSR-infinitives is considered to be trace copy (t) because it is a residue of movement. The symbol $t$ represents movement of the subject which is initially known as trace. The subject to-infinitives originates from the [spec-VP] To-infinitives answering the questions because the verb, answer, is a two-place predicate, meaning that there should be an agent to answer and something to be answered. After being merged with the AUX be, the D to-infinitives moves from the [spec-VP] leaving its trace-copy $\boldsymbol{t}$, and re-merges with the AUX-bar be $\boldsymbol{t}_{\boldsymbol{i}}$ answering the questions. To satisfy the T-feature, it further moves to the lower [spec-TP] leaving another copy to check the EPP feature which attracts the closest noun expression to become a subject (Radford, 2009). Finally, the D to-infinitives is attracted by the EPP feature carried by the finite T $\varnothing$ forming the TP To-infinitives appear $\boldsymbol{t}$ to $\boldsymbol{t}$ be $\boldsymbol{t}$ answering the questions. The co-indexing illustrated in example (5) indicates the connectivity between the trace copies and the higher D to-infinitives. Just like the control infinitives, the lower copies of the D toinfinitives are deleted at the PF, and the syntactic elements that are only pronounced are toinfinitives, appear, to, be, answering, the, and questions.

Examples (4) and (5) reveal that the control and SSR-infinitives may have similar syntactic positions for their subjectness. In this case, there is connection between $\boldsymbol{t}$ and $\boldsymbol{P R O}$ because they originate from the same position which is the [spec-VP] and moved in a successive cyclic manner leaving their copies until they moved to the [spec-TP] in the matrix clause to check nominative case as well as the EPP feature. Interestingly, the paper further shows that both the control and SSR-infinitives are formed in a similar fashion, that is, through movement as maintained by Hornstein (1999) and Hornstein \& Polinsky (2010). Following Boeckx et al. (2010) on the notion of keeping theoretical apparatus constant, this study therefore, uses the trace-copy (t) instead of PRO of the control infinitives for consistency. The reason being, PRO is not base generated in this paper, but also a residue of movement.

\subsection{Movement Theory of Control}

The researcher indicated that the type of the subjectness for both the control and SSR-infinitives is generated in a similar fashion which is movement. This view is supported by the Movement Theory of Control (MTC) which states that control is like a raising in that the dependency between the 
controller and the controlee is facilitated by movement (Hornstein \& Polinsky, 2010). The generation of control infinitive is illustrated in example (6).

(6) [TP [D I VP [V attempt TP [DP PRO [T to VP [DP PRO [V answer [DP these questions]]]]]]]

(The structure is adapted from Radford, 2009; Hornstein, et al. 2010)

Example (6) demonstrates the PRO-free version of the MTC. The given example proves that the subjectness of control infinitive is generated through movement, and that its position is at the [specTP]. This result conforms to Nakagawa's (2001) reflection which reveals that PRO, the subject of the control infinitives is distributed at the [spec-TP]. However, this paper departs from Nakagawa's (2001) view that the subject PRO is positioned at the [spec-TP] to check the null case. The present research proposes that movement of the subject of the control infinitives from the [spec-VP] is triggered by the EPP feature leaving out its copies in a form of PRO. Therefore, the specifier $I$ in example (6) is the controller of PRO that first became the subject of the V answer, then moved to [spec-TP] leaving its copy, PRO to become a subject of T to. From the lower [spec-TP] it finally moves to the matrix clause subject position leaving its copy PRO. The evidence that the specifier $I$ is the controller of the PRO approves the idea posited by Radford (1997) and Chomsky (2015) that PRO is controlled by its antecedent.

The findings in the paper further demonstrate that the distribution of the control subject is similar to that of the SSR-infinitives, and that DP bears multiple $\theta$-roles (Hornstein \& Polinsky, 2010). The location of the subjectness of the control infinitive is illustrated in example (7). The hierarchy is adapted from Hornstein, 1999; Hornstein \& Polinsky (2010).

(7) [IP [D We VP [V hope $\mathrm{vP}$ [D we [v to VP [D we [V represent [PP to ourselves]]]]]]]

Example (7) reflects the syntactic structure which indicates the subjectness of the control infinitive we hope to represent to ourselves. This research demonstrates an interesting result that the subjectness of the control infinitive is a lexical item as seen in example (7) that D we is the subject of the control infinitive to represent to ourselves, and it is positioned at the [spec-vP]. The study adopts Nakagawa's (2001) observation that the location of the subjectness of control infinitive must be at [spec-vP] of the infinitive clause. Nonetheless, the study departs from it with regard to the type of subjectness of the control infinitives which is PRO. This study reveals that the subjectness of the control infinitives can also be a lexical item because the PRO-free MTC rejects the $\theta$-criterion principle by permitting the DP to bear multiple $\theta$-roles, and allows movement to $\theta$-positions (Boeckx et al., 2010; Hornstein \& Polinsky, 2010).

The paper also shows syntactic elements which were selected from the numeration \{we, hope, to, represent, to, ourselves $\}$. PRN ourselves is merged with preposition (P) to forming prepositional phrase (PP) to ourselves. V represent is also selected, and merged with the PP to ourselves. A resulting constituent is V-bar represent to ourselves. $\bar{V}$ is further merged with D we to form VP we represent to ourselves. The specifier we subsequently merges with verbaliser (v) to in order to form $v$-bar to we represent to ourselves. Since the $\boldsymbol{v}$ hosts the external argument (Čakányová, 2018), the $\mathrm{D} \boldsymbol{w} \boldsymbol{e}$ is attracted to the $\boldsymbol{v}$ position leaving its copy at the [spec-VP], and re-merges with the $\mathrm{v}$ to $\boldsymbol{w e}$ represent to ourselves to form verbaliser phrase $(\mathrm{vP})$ we to we represent to ourselves. Following $\mathrm{Al}-$ Horais (2013), it should be noted that move operation left behind the copy of the D we. Another V hope is selected and merged with the $\mathrm{VP}$ we to we represent to ourselves to form VP hope we to we represent to ourselves. To satisfy the EPP feature carried by the inflection (I), D we is moved to the [spec-IP] leaving another copy $\boldsymbol{w e}$ at the [spec-vP] and re-merged with the I-node. The resulting constituent is the IP we hope we to we represent to ourselves. 
A further finding is that the type of the subjectness of the control and SSR-infinitives is identical, and the position is still the same as shown in example (8) a. and b.

(8) a. [IP [D $I_{i} \mathrm{VP}\left[\mathrm{V}\right.$ plan $\mathrm{vP}\left[\mathrm{D} \boldsymbol{t}_{i}\left[\mathrm{v}\right.\right.$ to $\mathrm{VP}\left[\mathrm{D} t_{i}[\mathrm{~V}\right.$ test [DP the two competing hypothesis]]]]]]] (The hierarchy is adapted from Boeckx et al., 2010)

b. [IP [D Readers $s_{i}\left[\mathrm{I}\right.$ are AP [A likely $\mathrm{vP}$ [D $\boldsymbol{t}_{i}\left[\mathrm{v}\right.$ to VP [D $t_{i}$ [V make [D claims]]]]]]]] (The hierarchy is adapted from Boeckx et al., 2010)

The analysis in example (8) a. and b. signal the subjectness of the control and SSR-infinitive embedding clauses correspondingly. The results in the study show that the subjectness of both the control and SSR-infinitives is the trace-copy $(t)$, and the subject in both structures is positioned at the [spec-vP]. The reflection on the type of the subjectness of both the control and SSR-infinitives does not support the previous literature such as Kučanda's (2000), Nakagawa's (2001), and Runner's (2006) studies. Their results stipulate that the subjectness of the control infinitives differs with that of the SSR-infinitives. However, the present study noted that the subjectness of the SSR and control infinitives are the same as their structures are generated in a similar fashion. In line with the example (8) a. and b., their subjectness trace-copy conforms to Boeckx et al.'s (2010) claim that the MTC was incompatible with previous theories that assumed the Deep Structure (DS), but the elimination of the DS has let to new analysis of the subjectness of the control infinitives.

\subsection{External Token Merge}

The current study reflects that the subjectness of the subject-less conventional infinitives is the lexical item. Krivochen (2012) asserts that instead of moving an element, another instance can be drawn from array. A similar syntactic element can be drawn as many times as possible to satisfy the conceptual-intentional system. The use of the External Token Merge (ETM) leads to the elimination of the PROs. The claim is maintained by Krivochen \& Kosta (2013) that the ETM comprises array with extra copies. According to Krivochen (2012) and Krivochen \& Kosta (2013), array is a set of types of units identified at the semantic interface, and from these types, infinite number of tokens which are also known as instances could be realised. The finding drawn from the control infinitives is demonstrated in example (9).

(9) [TP [D We VP [V try TP [D we [T to VP [D we [V front [DP the particle]]]]]]] (The hierarchy is adapted from Boeckx et al., 2010)

Example (9) shows the structure in which the subjectness of the control infinitive we try to front the particle is identified. From this example, the finding demonstrates that the subjectness of control infinitive is a lexical item positioned at the [spec-TP]. The subjectness of to front the particle is D we located at the [spec-TP]. The observation in this paper does not conform to the findings in the reviewed previous studies such as Kučanda's (2000), Kalane's (2007) and Smouse's (2010) studies because they view PROs as the subjectness of the control infinitives not the lexical items. They view the subjectness of control infinitives as construal. That is, a base generated syntactic element, PRO. Nonetheless, the present study emphasises that the subjectness of the control infinitives is a lexical item that has many instances in the array to fill thematic domain as also observed by Krivochen (2012) and Krivochen \& Kosta (2013). The paper also shows the following lexical items from the array in example (9): $\{\boldsymbol{w e}$, try, to, front, the, particle $\}$. However, the D we has three instances from array as seen in example (9). This reflection is attested to by Krivochen (2012) who notes that the 
occurrences of we are different tokens of the same type at the LF. Instead of movement, D we is merged with V-bar front the particle, and the resulting constituent is VP we front the particle. Another instance of the D we is selected and externally merged with an intermediate projection $\overline{\mathrm{T}}$ to we front the particle. The last instance of the $\mathrm{D}$ we is selected from the array and merged with null $\mathrm{T}$ to form $\mathrm{TP}$ we try we to we front the particle. The merging of the $\mathrm{D}$ we is done to satisfy the concept of theme.

Another evidence showing the position of subjectness of the subject-less conventional infinitives was drawn from the SSR-infinitives as shown in example (10). The hierarchy is taken from Radford (2009)

(10) [TP [DP The writer [T ought VP [DP the writer [V ought TP [DP the writer [T to AUXP [DP the writer [AUX have VP [DP the writer [V elaborated PP [P on [DP the first clause]]]]]]]]]]]

Example (10) reflects the structure in which the subjectness of the SSR-infinitive is revealed. The results indicate that the subjectness of the SSR-infinitives is the lexical item which is positioned at the [spec-TP]. This finding is supported by Boeckx et al. (2010) that the type and the location of the subjectness of the SSR-infinitives are similar to that one of the control infinitives, and that they are created in the embedded subject position. The DP the writer has five instances that are selected and first merged with the lower V-bar elaborated on the first clause, AUX-bar have the writer elaborated on the first clause, the lower T-bar to the writer have the writer elaborated on the first clause, the higher V-bar ought the writer to the writer have the writer elaborated on the first clause and the higher T-bar ought the writer ought the writer to the writer have the writer elaborated on the first clause correspondingly. The resulting constituent is TP the writer ought the writer ought the writer to the writer have the writer elaborated on the first clause. These five instances of the DP the writer are selected for thematic reasons. As for ought, it is just selected and merged with the lower TP to occupy the $\mathrm{V}$ position and moves from the $\mathrm{V}$ position for syntactic reasons to occupy the $\mathrm{T}$-node as it is one of the auxiliaries that occupy the T-node, hence raising. Based on the distribution of the subjectness of the subject-less conventional infinitives, the findings in this sub-section are supported by Nakagawa (2001), who states that starting from the ME, the subjectness of the conventional infinitives is distributed at the [spec-TP].

Another position of the subjectness of the subject-less conventional infinitives noted in this paper is the embedded infinitives whose syntactic structures are the IPs. This incidence that shows the structure of the subjectness of the control infinitive is illustrated in example (11).

(11) [IP The student to [VP The student persuade the reader [IP The reader to [VP The reader accept this conclusion [IP the student [VP The student has [IP The student to [VP The students show [IP what [VP made him [IP [VP come to such conclusion]]]]]]]]]]] (The hierarchy is adapted from Polinsky \&Potsdam, 2006)

Example (11) illustrates the structure which portrays the subjectness of the control infinitive to persuade the reader to accept this conclusion, the student has to show what made him to come to such conclusion. The observation drawn from the given data shows that the subjectness of the control infinitives can be at [spec-IP]. The finding goes beyond Rashid's (2017) findings as they do not state the position of the subjectness of the control infinitives, but the paper reflects the distribution of the subjectness which is the [spec-IP]. An interesting observation is that the clauses in some IP structures like the one in example (11) is headed by the IPs. In this paper, it is illustrious that the type of the subjectness of the control infinitives is also a lexical item. From example (11), the subjectness of subject control infinitive to persuade the reader is DP the student. For this type 
of infinitive, its subjectness is controlled by the controller the student in the matrix clause the student has. As for the object control infinitive to persuade the reader to accept this conclusion, its subjectness is DP the reader while the subjectness of the SSR-infinitive to show what made him to come to such conclusion is DP the student. DP the student has six different tokens of the same type, the student. They are different tokens because they occupy different $\theta$-roles positions. Different from the DP the student, the DP the reader has three instances from the array. It is worth noting that within the SSR-infinitive, the student has to show, there is an embedded SOR-infinitive what made

him to come to such conclusion of which its subjectness is not interpreted since it is not the concern of the current paper. Despite the complexity of this embedded infinitive clause which has a recursion feature, the distribution of the subjectness of all the subject-less conventional infinitive embedded clauses is at the [spec-IP].

Tapping from the SSR-infinitives, its subjectness is also a lexical item which is located at the [specIP]. The incidence is shown in example (12).

(12) [IP Scripts [VP (Scripts) appear [IP Scripts to [VP Scripts be the cognitive structures]]] (The structure is taken from Polinsky and Potsdam, 2006)

Example (12) reflects the structure that entails the subjectness of the SSR-infinitive scripts appears to be the cognitive structures. The results from the given data reveal that the subjectness of the SSRinfinitives is distributed at the same position as the control infinitives which is at the [spec-IP] (Hornstein, 1999); Polinsky and Potsdam (2006). The type of the subjectness that the SSRinfinitives have is also the lexical item. The DP Scripts originates from the lower [spec-VP] where it checks for $\theta$-role. The DP Scripts is also selected and merged to occupy the [spec-IP] in order to check for $\theta$-role. Unlike the MTC analysis, another DP Scripts is selected and merged to occupy the higher [spec-VP] for thematic reasons. Contrary to the thematic considerations, the DP Scripts positioned at the higher [spec-IP] is merged to check the D-feature of the IP (Hornstein, 1999). This the present paper further indicates that the subjectness of the subject-less conventional infinitives is a lexical item that bears multiple $\theta$-roles while those for the predecessors, the subjectness of the control infinitive is different from that of the SSR-infinitives. This study divulges that they are the same. Furthermore, the previous analyses were grounded by the theta criterion principle which states that one argument bears one theta role. The current paper notes that not only the syntactic features attract DPs to the specifier positions, but also the semantic requirement such as thematic requirements as maintained by Krivochen and Kosta (2013).

\section{Conclusions}

The aim of this paper was to describe the position of the subjectness of the subject-less conventional infinitives. I assumed that the subjectness of the subject-less conventional infinitives appears before the infinitive-to in a sentence. Based on the reflection in the present paper, the assumption seemed not to be supported as it does not give the exact syntactic position of the subjectness of the subjectless conventional infinitives; it just states that it appears before the infinitive-to. The results from the evidence reveal that the subjectness of the subject-less conventional infinitives can be located at the [spec-IP], [spec-TP], and [spec-vP]. The paper therefore, concludes that the distribution of the subjectness of the subject-less conventional infinitives varies depending on the structure of the subject-less conventional infinitives. Meaning that if the structure of the subject-less conventional infinitives is the IP, the subjectness will be at the [spec-IP]; if it is the TP, the subjectness must be positioned at the [spec-TP]; and if the structure is the vP, the subjectness should be located at the [spec-vP]. This positioning of the subjectness of the subject-less conventional infinitives is visible 
at the LF. Based on this finding, the paper concludes that the type of the subjectness of the control and SSR-infinitives is the same. They can have a trace-copy as their subjectness as they are generated through movement. They can also have the lexical subjects not the null ones as the MTC and ETM permit one argument to bear multiple roles.

\section{References}

[1] Al-Horais, N. (2013). The Minimalist Program and its New Insight to the Concept of Universal Grammar. Journal of Universal Language, 14(2), 79-112.

[2] AlTameemy, F.A., \& Alrefaee, Y.( 2021). Impact of Covid-19 on English Language Teaching in Yemen: Challenges and Opportunities. TESOL International Journal. 16, 238-252.

[3] Boeckx, C., Hornstein, N. and J. Nunes. (2010). Control as Movement. Cambridge: Cambridge University Press.

[4] Chomsky, N. (2015). The Minimalist Program (20 $0^{\text {th }}$ ed.). Cambridge: The MIT Press.

[5] Davies, W.D. and S. Dubinsky. (2004). The Grammar of Raising and Control: A Course in Syntactic Argumentation. Malden: Blackwell Publishing.

[6] Graddol, D., Chesire, J. and Swann, J. (2006). Describing Language (2 ${ }^{\text {nd }}$ ed.). New York: Open University Press.

[7] Hornstein, N. (1999). Movement and Control. Linguistic Inquiry, 30(1), 69-96.

[8] Hornstein, N. and M. Polinsky (eds). (2010). Movement Theory of Control. Philadelphia: John Benjamins.

[9] Kibort, A. (2006). On Three Different Types of Subjectlessness and how to Model them in LFG. United Kingdom: University of Surrey.

[10] Kinsella, A.R. (2009). Language Evolution and Syntactic Theory. Cambridge: Cambridge. University Press.

[11] Krivochen, D.G. (2012). Internal Merge Beyond Explanatory Adequacy: [s.1.]: [s.n].

[12] Krivochen, D.G. and P. Kosta. (2013). Eliminating Empty Categories: A Radically Minimalist View on their Ontology. (05/03/2018). Retrieved from https://www.researchgatr.net/publication/316181465.

[13] Kučanda, D. (2000). Infinitive Subjects or Subject-less Infinitives". SL, 49(50), 81-9.

[14] Nakagawa, N. (2001). Bare vP Analysis of the Infinitival Clause in OE: Historical Development of Tough Constructions. English Linguistics, 18(2), 507-535.

[15] Omo, I.C. and Destiny, I. (2020). Pragmatic Hedges in Editorials: A Focus on Vanguard, Guardian and Sun Newspaper Editorials in 2017. Journal of Translation and Language Studies, 1(1), 26-36.

[16] Polinsky, M. and E. Potsdam. (2006). Expanding the Scope of Control and Raising. Syntax, 9(2), 171-192.

[17] Radford, A. (1997). Syntax: A Minimalist Introduction. Great Britain: Cambridge University Press.

[18] Radford, A. (2004). Minimalist Syntax: Exploring the Structure of English. Cambridge: Cambridge University Press.

[19] Radford, A. (2009). An Introduction to English Sentence Structure. New York: Cambridge University Press.

[20] Rashid, B.N. (2017). Subjectless Sentences vs. Subjectless Clauses. Journals of Language Teaching and Research, 8(6), 1116-1125.

[21] Riazi, A.M. (2016). The Routledge Encyclopaedia of Research Methods in Applied Linguistics: Quantitative, Qualitative, and Mixed-methods Research. London: Routledge.

[22] Runner, J.T. (2006). Lingering Challenges to the Raising to Object Control Constructions. [s.l.]: University of Rocherster.

[23] Wurmbrand, S. (1998). Infinitives. University of Vienna: MIT Press. 